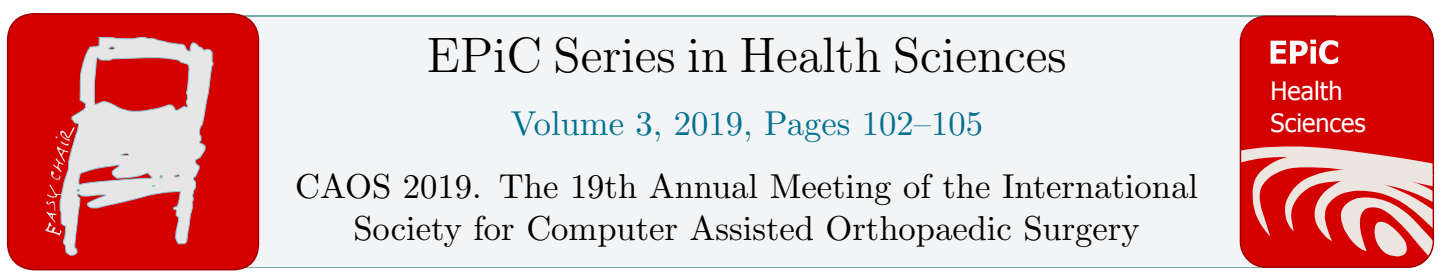

\title{
What to Expect in Short-term Outcomes of "Challenging” Patients with Computer-Assisted Total Knee Arthroplasty?
}

\author{
Yifei Dai $^{1^{*}}$, Quanjun Cui ${ }^{2}$, Michael P Bolognesi ${ }^{3}$, Samuel S Wellman ${ }^{3}$, \\ Thorsten Seyler ${ }^{3}$, Yassaman Najmabadi ${ }^{1}$, Charlotte Bolch ${ }^{1}$, David Liu ${ }^{4}$ \\ ${ }^{1 *}$ Exactech Inc, Gainesville, FL 32653, USA \\ ${ }^{2}$ University of Virginia School of Medicine, Charlottesville, VA, USA \\ ${ }^{3}$ Duke Orthopaedics, Durham, NC, USA \\ ${ }^{4}$ The Gold Coast Centre for Bone and Joint Surgery, Queensland, Tugun, AU \\ yifei.dai@exac.com
}

\begin{abstract}
This study investigated if CAOS TKA cases in higher risk patients would impact the outcomes of surgery. An average of 14-month postoperative outcomes on 58 TKAs from a prospective multicenter study were analyzed. The patients were grouped into challenging and standard case groups according to the criteria of age, BMI, comorbidities, and alignment deformity. Both groups demonstrated significant postoperative improvement in all outcome measures. Compared to the standard patients, the challenging patients achieved significantly higher improvement after TKA in KSS Knee score, while demonstrating the same level of improvements in all other outcome measures. Similarly, the two groups generally exhibit equivalent postoperative outcomes. The data demonstrated consistent postoperative results by CAOS TKA irrespective of patient conditions.
\end{abstract}

\section{Introduction}

Since its introduction to total knee arthroplasty (TKA), computer-assisted orthopedic system (CAOS) has been proven to significantly improve implant alignment in the knee consistency compared to conventional instrumentation [1,2]. Numerous studies on the CAOS TKA have been published focusing on postoperative implant alignment, intraoperative parameters, and postoperative outcomes and survivorship [3,4]. Additionally, the benefits of CAOS TKA in the patients with increased risks has 
been published. However, most of these studies are case reports and single arm series focusing exclusively on extra-articular alignment deformity [5-7].

Studies on conventional instrumented TKA concluded patient factors such as advanced age, obesity, multiple comorbidities, and severe alignment deformity may increase the risk of worse postoperative outcomes and early failure [8-11]. To date, it remains unclear how the outcomes CAOS TKAs are impacted by the "challenging" cases, as compared to the "standard" CAOS TKAs. This study analyzed a case series to investigate if there is a difference between challenging and standard cases in terms of the clinical short-term outcomes.

\section{Materials and Methods}

With institutional review board-approval and patient's signed informed consent, a prospective multicenter, consecutive TKA case series was conducted by 5 surgeons in a total of 70 patients (72 knees). All cases were primary TKA with the assistance of a contemporary CAOS system. The two bilateral patients in this series had their knees operated separately with at least 90 days apart.

"Challenging" cases were defined from the series as having one or more of the following conditions: 1) age greater than 80 years, 2) BMI greater than 35,3$) 3$ or more comorbidities, 4) coronal deformity greater than $15^{\circ}$, and 5) preoperative range of flexion (ROM) less than $90^{\circ}$. The latest available postoperative visit that was categorized as 1 year and beyond (staring from 9-month postoperatively) were reviewed and compared between the standard and challenging case groups. Specific outcomes assessed were: Range of Motion (ROM), Knee Society Score (KSS, knee and function), Hospital for Special Surgery Knee Score (HSS), Oxford Knee Score (OKS), Knee Injury and Osteoarthritis Outcome Score (KOOS: Symptom, Pain, ADL, QOL), and patient satisfaction Visual Analog Scale (VAS 1-10, with 10 indicating the highest satisfaction). Statistical significance was defined as $\mathrm{p}<0.05$.

\section{Results}

Fifty-eight knees were available for the analysis with an average follow-up period of 14 month. A detailed breakdown of challenging $(\mathrm{N}=28)$ and standard $(\mathrm{N}=30)$ cases is presented in Table 1. A summary of postoperative outcomes in the standard and challenging groups is presented in Table 2. Preoperatively compared to standard patient, the challenging patients had significantly worse scores in KSS Knee and HSS, and near significant worse score in KSS Function. Both groups demonstrated generally equivalent outcomes postoperatively and achieved significant postoperative improvement in all outcome measures compared to the preoperative baseline ( $\mathrm{p}$ values $<0.001$ ). The challenging patients improved even more in KSS Knee score compared to the standard patients ( $\mathrm{p}=0.02$, Table 2 ). Although standard patients had a higher KSS function score compared to that of the challenging patients $(\mathrm{p}=0.009)$, the challenging patients exhibited equivalent postoperative KOOS ADL $(\mathrm{p}=0.948)$ and generally higher satisfaction (VAS: $9.1 \pm 1.4$ ) compared to the standard patients (VAS: $8.8 \pm 2.2$ ) (N.S.). No device-related outstanding adverse events or revision was found in both groups at the time of analysis.

\section{Discussion}

With a previous study proving consistent accuracy using CAOS in TKA performed by surgeons of varying experience levels [12], this investigation sought to assess the sensitivity of the short-term postoperative outcomes of CAOS TKA to patient conditions. Although the conditions used to determine 
challenging cases in this study (age, BMI, number of comorbidity, degree of alignment deformity) have been shown by previous clinical investigations to negatively impact conventional TKA results [6-9], the present data did not suggest inferior postoperative outcomes should be in general expected in the challenging cases using CAOS. This may be due to the benefits of CAOS facilitating intraoperative surgical guidance to help the surgeon mitigate surgical challenges and uncertainties while operating on the challenging patients, as well as avoiding intramedullary instrumentation. Like previous case studies and clinical series that have proposed the benefits of CAOS in TKA cases with severe coronal deformity [5-7], this study highlights the advantages CAOS TKA may offer in demanding cases where patient or joint factors increase the surgical challenges.

\begin{tabular}{lccc} 
A & & & \\
\hline & Challenging Patients & Standard Patients & $\mathrm{P}$ \\
$\mathrm{N}$ & 28 & 30 & - \\
Age (years, mean, range) & $67.0 \pm 10.5,41-82$ & $66.0 \pm 8.2,51-78$ & 0.687 \\
Female (\%) & 57 & 43 & 0.066 \\
BMI (mean, range) & $34.2 \pm 6.4,23.5-47.0$ & $29.9 \pm 2.5,25.0-34.9$ & 0.001 \\
Follow-up period (months) & $14.5 \pm 5.2,9-24$ & $14.3 \pm 4.4,9-23$ & - \\
\hline
\end{tabular}

$\underline{B}$

\begin{tabular}{lc} 
B Challenging Patients & $\mathrm{N}^{\dagger}$ \\
Age $>80 \mathrm{yr}$ & 4 \\
$\mathrm{BMI}>35$ & 22 \\
Coronal Deformity $>15^{\circ}$ & 1 \\
ROM $<90^{\circ}$ & 10 \\
Num Comorbidities $>2$ & 10 \\
\hline
\end{tabular}

† Each difficult case had one or more listed conditions.

Table 1. A) Patient conditions. B) Distribution of conditions in the challenging TKA group.

\begin{tabular}{lccc}
\hline Improvement from Baseline & Challenging Patients & Standard Patients & $\mathrm{P}$ \\
HSS & $25.0 \pm 16.6$ & $26.6 \pm 25.3$ & 0.783 \\
OKS & $17.9 \pm 10.3$ & $16.0 \pm 10.2$ & 0.493 \\
KSS & & & \\
Function & $23.2 \pm 21.4$ & $28.3 \pm 22.1$ & 0.375 \\
Knee & $33.7 \pm 19.2$ & $20.2 \pm 20.7$ & 0.020 \\
KOOS & & & \\
Pain & $40.0 \pm 24.6$ & $39.3 \pm 23.3$ & 0.988 \\
Symptoms & $34.8 \pm 22.8$ & $25.6 \pm 23.4$ & 0.855 \\
ADL & $38.9 \pm 23.4$ & $32.7 \pm 22.0$ & 0.313 \\
QOL & $46.7 \pm 23.6$ & $44.0 \pm 27.9$ & 0.699 \\
\hline
\end{tabular}

Table 2. Summary of postoperative improvements for the challenging and standard TKA groups.

\section{References}

[1] Brin YS, et al. Int Orthop,35(3):331-9,2011.

[2] Hetaimish BM, et al. J Arthroplasty,27(6):1177-82,2012.

[3] Schnurr C, et al. Int Orthop. 2012;36(11):2255-60.

[4] Schnurr C, et al. Orthopedics. 2010; 13;33(7):474.

[5] Mullaji A, et al. J Arthroplasty. 2009;24(8):1164-9. 
[6] Bottros J, et al. J Arthroplasty. 2008;23(1):74-8.

[7] Kuo CC, et al. J Arthroplasty. 2011;26(6):976.e17-20.

[8] Krushell RJ, et al. J Arthroplast 2007;22(6 suppl 2):77-80.

[9] Foran JR, et al. J Bone Jt Surg [Am] 2004;86-A(8):1609-15.

[10] Smith ID, et al. J Bone Joint Surg Br. 2008;90(11):1435-40.

[11] Elmallah RD, et al. J Knee Surg. 2015;28(5):411-6.

[12] Dai Y, Jung A, et al. CAOS 2017. Aachen, DE. 